\title{
Multilayer Traffic Engineering for GMPLS-Enabled Networks
}

\author{
Martin Vigoureux and Bela Berde, Alcatel Research \& Innovation \\ Loa Andersson, Acreo (Sweden), and Tibor Cinkler, BUTE (Hungary) \\ Lieven Levrau, Marconi Ondata, and Didier Colle, IMEC
}

Juan Fdez-Palacios, TID

Monika Jäger, T-Systems

\begin{abstract}
In recent years, significant work has been completed on traffic engineering enhancements to the generalized multiprotocol label switching protocol suite [1-3]. As a next step, reproducing the current trend of switching layers' integration happening in the data plane, network control is foreseen to go beyond the traditional per layer approach and tend toward an integrated model $[4,5]$. In these multilayer environments, a single GMPLS control plane drives various distinct switching layers at the same time and as a coherent whole, taking benefit from the "common" property of GMPLS. Beyond this application of supporting network control across different technologies, in this article we catalog the unified traffic engineering paradigms, discuss their applicability, and present their enforcement techniques. Furthermore, we show that the common GMPLS concept has the advantage of low operational complexity, and enables unified TE capabilities such as efficient network resource usage and rapid service provisioning.
\end{abstract}

\section{INTRODUCTION}

Generalized multiprotocol label switching (GMPLS) will enable unified traffic engineering (TE) for multilayer networks; that is, the same set of tools and the same paradigms are used for TE on all network layers. Current practice among incumbent operators is mostly to perform $\mathrm{TE}$ and network engineering (NE) centralized and offline. The activities are performed per network technology, independent of similar activities on other layers. The initial network resource provisioning and service configuration is derived from a sophisticated network planning and optimization process.

Network configuration, topology, and resource status are available in a database that is continuously updated and used for service provisioning. As services are being set up and torn down, there is a risk of resources being fragmented. To alleviate this, activities to free up resources are necessary and need to be undertaken carefully in order to avoid service disruption.

One drawback of the centralized approach is that it is much slower than actions taken by a control plane. The management system must access each node that participates in a path, and reserve resources and configure connectivity. Distributed control plane functions allow the automation of these processes with reduced delay and increased scalability. The dynamically switched network is an important part of the carriers' vision of future networking since it gives them a way to decrease costs and improve revenue.

The first stage of deployment has been under way for some time in networks with packet switch capable (PSC) devices only. We also see two other steps being taken, but in different directions. One step involves deploying networks with multiple switching capabilities, for example, PSC and lambda switch capable (LSC), each controlled by a control plane of its own. The other is deploying networks with multiple switching capabilities and one common control plane. Multiple switching capabilities could be present in a single node, or there could be just one switching capability per node.

Packet switching networks and circuit switching networks are today operated over the same physical network infrastructure (e.g., wavelengths). Frequently the transmission protocol stacks are disparate; say, synchronous optical network/synchronous digital hierarchy (SONET/ $\mathrm{SDH}$ ) or Ethernet. This creates unique opportunities and challenges for novel control plane and TE enhancements in network architectures with switching on multiple layers. One objective is migration from per layer to an integrated mode of operation. A common control plane instance replaces the separate control plane instances per data plane switching layer. The information 


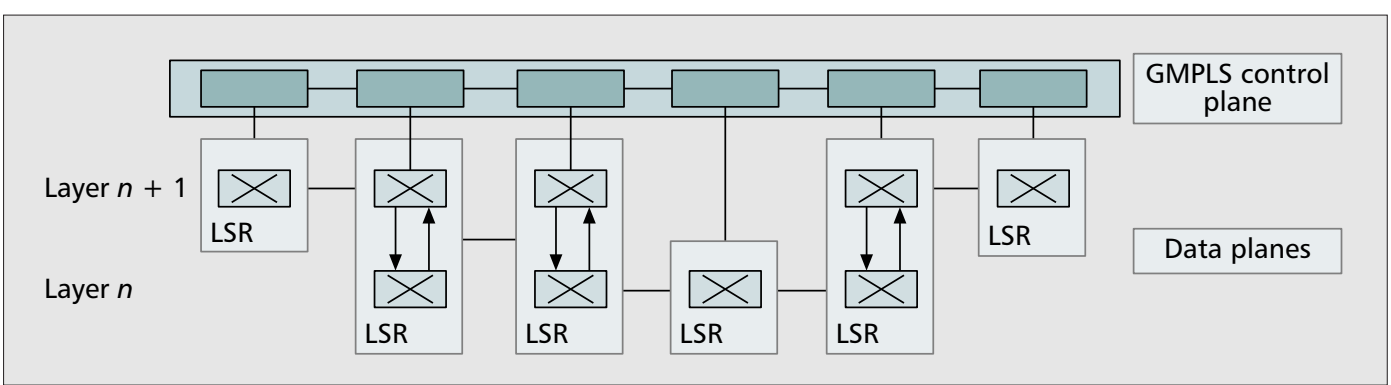

Figure 1. The general case of a network with switching capabilities on two layers.

databases created by the common control plane are also used for unified TE.

GMPLS is a way to integrate the network control plane and central network management tasks. The goal is to introduce more dynamic TE functions based on efficient heuristics and managed TE policies so that resource management and policies to be applied can be harmonized in a scalable way for all network layers. One area where the benefits of this approach are obvious is in coordination of recovery actions between different layers; this is hardly possible in nonGMPLS networks, where the network layers perform recovery actions separately. This article is limited in scope and only covers the area controlled by one GMPLS control plane instance, for example, one autonomous system (AS). The global view of the status in the whole network area and its related TE information from different switching layers leads to more efficient resource utilization and network operation.

Different routes, depending on starting points, may be used to achieve this goal. Incumbent operators with several networking technologies might start by introducing separate control planes per layer. Green field operators with a simpler protocol may choose to go for the unified model directly.

The rest of the article is organized as follows. In the next section we present a brief outline of fundamental TE paradigms. Next, we describe the strategy we use to improve the efficiency and robustness of the unified TE features of a GMPLS control plane for multilayer network architectures. Last, we present TE enforcement techniques by means of policy-based management (PBM) mechanisms.

\section{Traffic ENGINEERING CONCEPTS}

This section gives an overview of multilayer TE terms and concepts relevant for the discussion in this article.

\section{MULTILAYER ENVIRONMENTS}

Networks are often partitioned into different domains (e.g., an AS). In a multilayer environment such a network partition will have equipment with switching capabilities on multiple layers. Label switching routers (LSRs) in multilayer networks may have different sets of interface switching capabilities (ISC). The example below and Fig. 1 show a two-layer network, with switching capabilities on the layers called layer$(n)$ and Layer- $(n+1)$. An LSR could be:

- Layer- $(n)$ switch capable
- Layer- $(n+1)$ switch capable

- Both layer- $(n)$ and layer- $(n+1)$ switch capable

In general, there is a mixture of the three cases, as shown in Fig. 1.

GMPLS control planes in multilayer networks controlled by a common GMPLS control plane are "unified" if the LSRs are either layer$(n)$ or layer- $(n+1)$ switch capable and "intelayer- $(n+1)$ switch capable. [1].

\section{TRAFFIC ENGINEERING AND Network ENGINEERING}

Network engineering deals, for example, with design, modeling, and planning of network resources and capacity (i.e., defining the resources needed). TE deals, for example, with efficient and reliable network operations while simultaneously optimizing network resource utilization and traffic performance in operational networks (i.e., allocating resources where they are needed).

There are several TE approaches that deal with traffic demand variations, traffic performance, resource optimization, and failure scenarios in a network. TE has become an indispensable function in many large networks because of the high cost of network resources, and the commercial and competitive nature of modern networking. To achieve the TE goals these operations should be performed without any loss of the quality of service (QoS) connection requirements. The optimization aspects of TE can be achieved through capacity management and traffic management, while traffic performance can be improved through performance monitoring and a combination of analytical and empirical methods. Thus, the control dimension of TE responds at multiple levels of temporal

One way to categorize TE is in time-dependent, state-dependent, and event-dependent methods. Time-dependent TE algorithms are applicable to predictable traffic variations, such as daytime peak hours or a shift from business to residential traffic in the evening. In those cases the QoS requirements and traffic matrix may be roughly estimated in advance. State-dependent TE methods use the current state of the network to adapt to variations in actual traffic. Constraint-based routing is one example of state-dependent TE operations. Event-dependent TE methods are adaptive and distributed in nature. One example is the responses to link and node failures. grated" if all LSRs are both layer- $(n)$ and resolution to network events.
TE has become an

indispensable

function in many

large networks

because of the high

cost of network

resources and the

commercial and

competitive nature

of modern

networking. To

achieve the TE goals

these operations

should be performed

without any loss of

the QoS connection

requirements. 


To coordinate the TE
capabilities of each
layer in a multilayer
network a key factor
is that the NE is
aware of the
multilayer
characteristics.
Working on longer
time scales, using
knowledge of traffic
trends and variations,
NE is capable of
computing target
network states.

To coordinate the TE capabilities of each network a key factor is that the NE is of the multilayer Working on longer time scales, using rends and variations, capable of network states. (a)
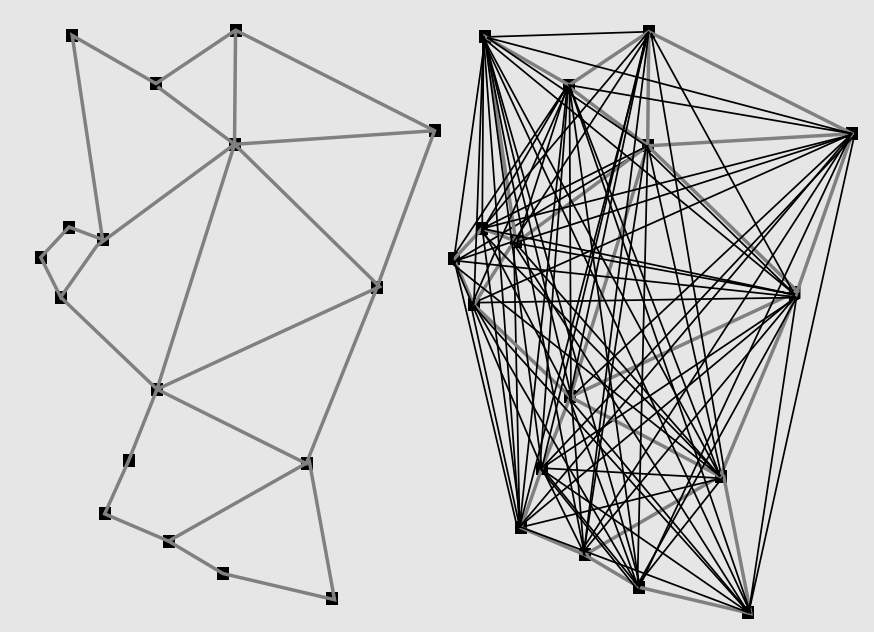

(c)

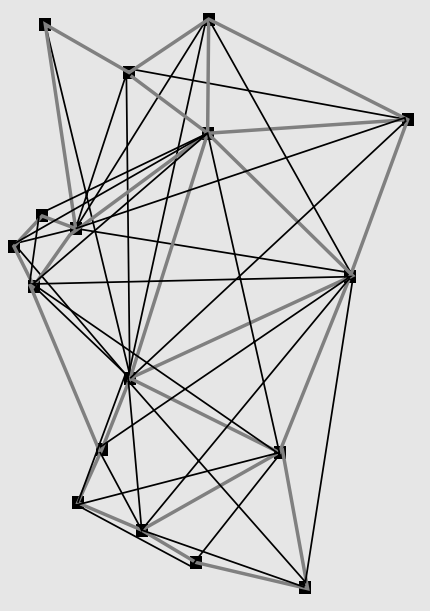

Figure 2. Virtual topologies: a) one-hop transport LSPs; b) full mess of transport LSPs; c) optimized mesh of transport LSPS.

TE and NE can be both proactive and reactive. In the first case the network tries to maintain an efficient state. In the latter case actions are only taken when certain inefficiency conditions are detected (e.g., exceeding a threshold).

In TE these actions may be performed on existing connections or apply to new connection requests. In the former case, connections may be temporarily interrupted or QoS degraded. In the latter case, it may take a long time before an undesirable network state is resolved and the connection is established. In an operational network with strong TE requirements both methods need to be used.

To coordinate the TE capabilities of each layer in a multilayer network, a key factor is that the NE is aware of the multilayer characteristics. Working on longer time scales, using knowledge of traffic trends and variations, NE is capable of computing target network states. TE, working on shorter time scales, may use theses pre-computed network states to achieve a flexible and scalable resource and traffic optimization.

\section{Grooming TeCHNIQUeS}

Deployment of multilayer networks brings new challenges. Switching capabilities on multiple layers makes it possible to control, operate, and traffic engineer a network in new ways. TE is no longer limited to tackling just the traditional path computation process and setting up the path the traffic flow will follow. Routing decisions must be made considering all dynamic layers involved in the transport of the flow, including at what points cross-layer operations should be performed. This goal is greatly facilitated by the visibility of all layers and the TE information related to these layers.

Directly linked to the transport of traffic flows across multiple layers is the grooming paradigm. To aggregate multiple traffic streams, sharing a common path, into one or more label switched paths (LSPs), and later separate them is referred to as traffic grooming. The set of connections on a given layer dynamically established for grooming purposes serves as an abstract or virtual topology (VT) for the upper layer. This type of grooming we consider adaptive grooming. Adaptive grooming refers to the case where the upper and lower layers are controlled by a single control plane instance; a new demand at any layer has influence on other layers as well (e.g., the routing decision has an effect on multiple layers).

Grooming addresses the TE issue of finding a trade-off between the lower cost of switching on a lower layer and the better resource utilization in switching on higher layers. In an integrated layer 2 over layer 1 network, with network elements that provide layer 2 and layer 1 switching, there are two extreme cases, and a trade-off needs to be found:

- The VT matches the physical topology (Fig. 2a).

- The VT interconnects the layer 2 switches in a full mesh (Fig. 2b).

The drawback in the first case is a relatively high number of layer 2 hops, because fiber connectivity generally is a scarce resource. The drawback in the second case is that layer 1 resources will be wasted.

\section{RESILIENCE CONSIDERATIONS}

While TE optimizes the use of resources under normal operation, resilience mechanisms such as protection, restoration, reroute, and recovery are TE mechanisms that are invoked in case of single or multiple failures. These mechanisms are also used to switch traffic back to resources when they come back up after being repaired.

The resilience techniques defined for singlelayer networks can be used for multilayer networks as well [6, 7]. Resilience for multilayer networks raises issues regarding coordination between layers during recovery operations. One of the most critical issues is to decide which recovery action needs to be taken on which layer. Following is a nonexhaustive list of the parameters that play a role in recovery decisions: - Mode (protection or restoration) 
- Scope (end-to-end or segment-based)

- Scheme (1+1, shared mesh, etc.)

- Risk (Shared Risk Link Group, SRLG, diverse)

- Loss of connectivity (from loss of light to loss of routing adjacency)

Each layer has the capability to detect failures within that layer and sometimes also trigger resilience actions. One critical aspect of resilience actions to be taken into account in a multilayer network is that they have to be carefully coordinated between layers.

\section{APPLICABILITY TO GMPLS ENVIRONMENTS}

\section{GMPLS KEY TE BUILDING BLOCKS}

In this section we introduce the key GMPLS TE building blocks.

TE Link - A TE link is a link between two adjacent LSRs that has a set of associated TE capabilities. A TE link can consists of a number of bundled component TE Links, which can themselves recursively comprise bundled TE Links. Free and allocated link capacities and TE attributes of the link components are represented in an aggregated form.

Link Bundling - This is a construct that makes it possible to group individual TE links together and shows the available resources in an aggregated way. Link bundling improves the routing scalability by reducing the amount of information to be processed by the control plane. With link bundling a one-to-one association between a regular routing adjacency and a TE link is no longer required. Hence, the number of routing adjacencies in the network can be kept proportional to the number of control plane adjacencies and is not related to the actual number of data plane links.

Forwarding Adjacency - TE links have been extended to nonadjacent devices by introducing the forwarding adjacency (FA) concept. A GMPLS-capable node can advertise an LSP as a TE link into a single routing domain. Such a link is referred to as an FA, and the corresponding LSP as an FA-LSP. The routing protocol (Open Shortest Path first with TE, OSPF-TE, or Intermediate System to Intermediate System with TE, ISIS-TE) floods the link state information about FAs just as it floods the information about any other TE link. This allows other nodes to use FAs as any other TE link for path computation purposes. The use of FAs provides a mechanism for improving bandwidth utilization and enables aggregating forwarding states. FAs allow the creation of an LSP hierarchy and thus improve the scalability of GMPLS-capable control planes.

The TE extensions of the routing protocol such as OSPF-TE use so-called sub-type length values (TLVs) for specifying and distributing information of the TE links. In a multilayer environment important TE properties of a TE link are defined by one or more switching capabilities associated with it. GMPLS defines control plane capabilities for packet (PSC), layer 2 (L2SC), time-division multiplexing (TDM-SC), lambda (LSC), and fiber (FSC) switching capabilities. The related TE attributes of the switching capabilities are defined, for example, in [3].

Routing and signaling protocols may use the TE attributes to perform constraint-based path computation, optimizing the path on other constraints than least cost (e.g., resource consumption or available bandwidth).

\section{MULtILAYER ROUTING AND Signaling}

This section discusses signaling and routing issues and two methods of route computation in a multilayer network.

There are several possible methods for route computation in multilayer environments, and the methods could also be combined.

A full view of the state of all layers in the network and the TE information makes coordination between layers possible. The coordination leads to optimal routing, connection establishment, and signaling decisions. It also implies that the control plane needs to deal with an amount of information substantially larger than that for a comparable IP network. One consequence is that it will be more resource- and time-consuming to run routing algorithms. There are basically two ways to cope with this, a layered routing approach and a multilayer approach.

In the first mode, path computation is performed on a per layer basis, and the lower-layer information is abstracted in a VT the computing layer can use. In the second mode the knowledge of all layers is used to compute routes.

In the first mode a VT is created by the common control plane and consists of lower-layer FA-LSPs between the upper-layer nodes. The control plane uses the FA-LSPs to establish upper-layer routes.

As an example, assume that FAs instantiated by LSC LSPs provide a virtual network topology to a PSC layer. This topology of FAs provides all the TE information and enables TE routing at the PSC layer. An established PSC LSP is composed of links connected to equipment capable of switching this type of LSP only; that is, the LSP is routed on a single layer.

TE also plays an important role in multilayer signaling. GMPLS signaling (and routing) protocols adapt the VT to traffic demands (i.e., FALSPs could be set up or taken down as needed). When an LSP crosses the boundary from an upper to a lower layer, it may be nested in or stitched to a lower-layer FA-LSP. The decisions to establish or take down an FA-LSP could make use of thresholds enforced via PBM (see below) to avoid link state instability.

The second method takes advantage of having a complete view of all layers from a topological and TE resource standpoint. The route resulting from path computation will be multilayer in nature (i.e., it traverses links of different switching capabilities). This enables a path to be set up end to end across the different layers, where each successive LSP participates in the transport of the traffic flows.

Consider a PSC and LSC network transporting IP flows; an edge node could decide the route to traverse $[\mathrm{A}, \mathrm{B}, \mathrm{C}]$ at the PSC layer, then $[\mathrm{C}, \mathrm{D}, \mathrm{E}]$ at the LSC layer, and finally, $[\mathrm{E}, \mathrm{F}, \mathrm{G}]$
Each layer has the capability to detect failures within that layer and sometimes also trigger resilience actions. One critical aspect of resilience actions to be taken in a multilayer network is that they have to be carefully coordinated between layers. 


Without network
wide policies, there is
the risk that TE
decisions, for
example, on load
balancing, taken
locally by one LSR
may be inconsistent
with decisions taken
by other LSRs.
The PBM empowers
the operator to
coordinate
centralized and
distributed TE
processes.

at the PSC layer. For RSVP-TE signaling the identifiers of the links (used in the example) are more relevant than the nodes themselves. More sophisticated schemes may be envisaged (given more advanced node capabilities), such as imposing a cross-layer point between two routes in the LSC layer. This example shows the tight relationship between multilayer routing and multilayer signaling. By computing an explicit route across the different layers, the edge node may implicitly reques the triggering of LSP establishment in several layers in addition to specifying their explicit routes.

In the context of establishing multilayer LSPs between nodes with multiple ISCs or operating according to the integrated model raises a specific issue. To avoid unnecessary resource partitioning, nodes with multiple ISCs may advertise a single TE link with these multiple ISCs [3]. From that perspective the selection of a link in the route computation process needs to explicitly select a specific ISC among those advertised. Existing signaling protocols could be extended to disambiguate the indication of the ISC on multi-ISC links by defining a new subobject to RSVP-TE.

Taking advantage of both the FA paradigm and the global multilayer vision, a combination of the above two methods is expected to be the practical choice with regards to multilayer routing and signaling. Routing is dependent on the network topology and associated link states. Routing stability may be impaired by frequent changes in the VT or the status of links in the VT. Creation and deletion of LSPs may be triggered by adjacent layers or through operational actions to meet changes in traffic demand.

\section{TE ATTRIBUTES AND INHERITANCE}

The different TE attributes introduced above are expected to play a role in the path computation process. Path computation typically relies on running a shortest path algorithm over a weighted graph. Certain of these TE attributes could be used to construct a graph satisfying the requirements of the connection to establish. For example, TE links not having enough bandwidth could be pruned from the TE database and not appear in the graph. Other TE attributes could be used to assign a cost to the links of the graph. The TE metric, or a combination of TE metric and other attributes, could serve that purpose.

Currently the TE metric is defined on a TE link basis. In multilayer networks some TE links may have several switching capabilities. However, different switching technologies may have different constraints. While for circuit switching technologies the objective is most often to groom traffic as much as possible to optimize resource usage, in packet technologies load balancing is sometimes preferred to keep congestion and blocking probabilities low. When a single TE link contains several switching capabilities, a single TE metric cannot simultaneously tackle both packet and circuit specifics. This suggests a more flexible TE metric, allowing a TE metric per ISC, facilitating layer-specific path computation.

As mentioned previously, FAs enable optimization of preprovisioned or precomputed VTs but also dynamic triggering of resources at various ISCs. However, challenges arise from routing an advertisement of FA-LSPs as TE links. FAs consist of an abstraction of the underlying layer in which the FA-LSP has been established; thus, an FA will summarize the TE attributes of the spanned TE links.

The selection of a given FA link by the path computation process has implications for both the transported LSP and the layer in which the FA-LSP is established. The summarization or inheritance process should thus appropriately reflect the lower-layer information of interest to the upper layer. For example, from a connectivity point of view two FAs are identical (i.e., they interconnect two upper layer points). However, one might be two hops and the other six hops long in the underlying layer. The selection of one of these FAs and the potential dynamic triggering of additional bandwidth will have a clearly different impact on the transport layer. In addition, different FAs might differ in terms of delay and/or jitter for a packet layer. The TE metric could be used to differentiate these cases.

In multilayer networks the inheritance of protection and restoration related TE link attributes must also be considered. However, applying simple attribute inheritance might not be sufficient to distinguish different recovery schemes. It is impossible to differentiate a 1:1 end-to-end protected LSP advertised as a single FA together with its backup LSP from a single FA-LSP routed over links providing 1:1 protection. A link protection type of 1:1 would be used for the two corresponding TE link advertisements. An upper layer would thus have no means to differentiate the two cases. However, these two recovery schemes (end-toend and span) have major differences in terms of recovery delay and robustness.

\section{ENFORCING TE THROUGH Policy-BASEd MANAGEMENT}

A policy is a set of rules guiding the LSRs in making their local TE decisions. The TE policies are set by the operator and scope the degrees of freedom of the LSRs, and are intended to tune the overall network behavior according to the operational goals. Without network-wide policies, there is the risk that TE decisions, for example, on load balancing made locally by one LSR may be inconsistent with decisions made by other LSRs. The PBM empowers the operator to coordinate centralized and distributed TE processes.

TE policies guide both signaling and routing in a GMPLS network. In Fig. 3 the management abstraction and hardware abstraction layers hide the internal implementation details of the respective interfaces toward the network management and hardware. To name only a few, examples of TE policies for routing are:

- Whether or not to advertise a given LSP as a TE link, enabling the creation of VTs

- Criteria for bundling taking into account, for example, SRLG information for facilitating risk disjoint path computation over abstract topologies

Examples of TE policies related to signaling are: 
- Parameters involved in the setup of the working and protection LSPs

- Stitching (dedicated path segments) and nesting operations (shared path segments) in case of loose hop

- Strategies for triggering LSP setup

- Parameters involved in the recovery process, such as failure notification and reverting recovery operations

The PBM enforces conditions on an inherently distributed and completely autonomous GMPLS TE environment [8].

\section{CONCLUSION}

This article combines concepts from three areas of research. First, we show that the unified TE paradigm improves the accuracy of TE decisions in the multilayer network. It widens the applicability of well-known TE techniques to include different switching technologies. It enables network resource optimization across multiple network layers. Second, the unified TE is based on an extensible GMPLS protocol framework, and third, the PBM can be used to improve the accuracy of the TE operations through the use of operator policies.

Unified TE provides an alternative means for operators who need to reduce complexity and improve the operational and cost efficiency of their multilayer networks.

\section{ACKNOWLEDGMENT}

This work was carried out in collaboration between partners of the IST project NOBEL funded by the European Commission.

\section{REFERENCES}

[1] E. Mannie, Ed., "Generalized Multi-Protocol Label Switching (GMPLS) Architecture," RFC 3945, Oct. 2004.

[2] D. Katz et al., "Traffic Engineering (TE) Extensions to OSPF Version 2," RFC 3630, Sept. 2003.

[3] K. Kompella et al., "OSPF Extensions in Support of Generalized Multi-Protocol Label Switching," draft-ietf-ccamp-ospfgmpls-extensions-12.txt, Oct. 2003, work in progress.

[4] K. Shiomoto et al., "Requirements for GMPLS-Based MultiRegion and Multilayer Networks," draft-shiomoto-ccampgmpls-mrn-reqs-00.txt, Oct. 2004, work in progress.

[5] E. Dotaro et al., "Multi-Region Networks: Generalized Multi-Protocol Label Switching (GMPLS) as Enabler for Vertical Integration," Wksp. Proc. IEEE GLOBECOM, Dallas, TX, Dec. 2004, pp. 374-79.

[6] D. Papadimitriou and E. Mannie (Eds.), "Recovery (Protection and Restoration) Terminology for Generalized Multi-Protocol Label Switching (GMPLS)," draft-ietfccamp-gmpls-recovery-terminology-05.txt, Oct. 2004, work in progress.

[7] J.P. Lang and B. Rajagopalan, Eds., "Generalized MultiProtocol Label Switching (GMPLS) Recovery Functional Specification," draft-ietf-ccamp-gmpls-recovery-functional-03.txt, Oct. 2004, work in progress.

[8] B. Berde, et al., "Improving Network Performance through Policy-Based Management Applied to Generalized Multi-Protocol Label Switching," 10th IEEE ISCC 2005 Symp., June 2005.

\section{AdDitionAl READING}

[1] K. Kompella and Y. Rekhter, "LSP Hierarchy with Generalized MPLS TE," draft-ietf-mpls-Isp-hierarchy-08.txt Feb. 2001, work in progress.

[2] D. Papadimitriou et al., "Generalized Multi-Protocol Label Switching (GMPLS) Protocol Extensions for Multi-Region Networks (MRN)," draft-papadimitriou-ccamp-gmpls-mrnextensions-00.txt, Oct. 2004, work in progress.

[3] A. Banerjee et al., "Generalized Multi Protocol Label Switching: An Overview of Routing and Management Enhancements," IEEE Commun. Mag., Jan. 2001.

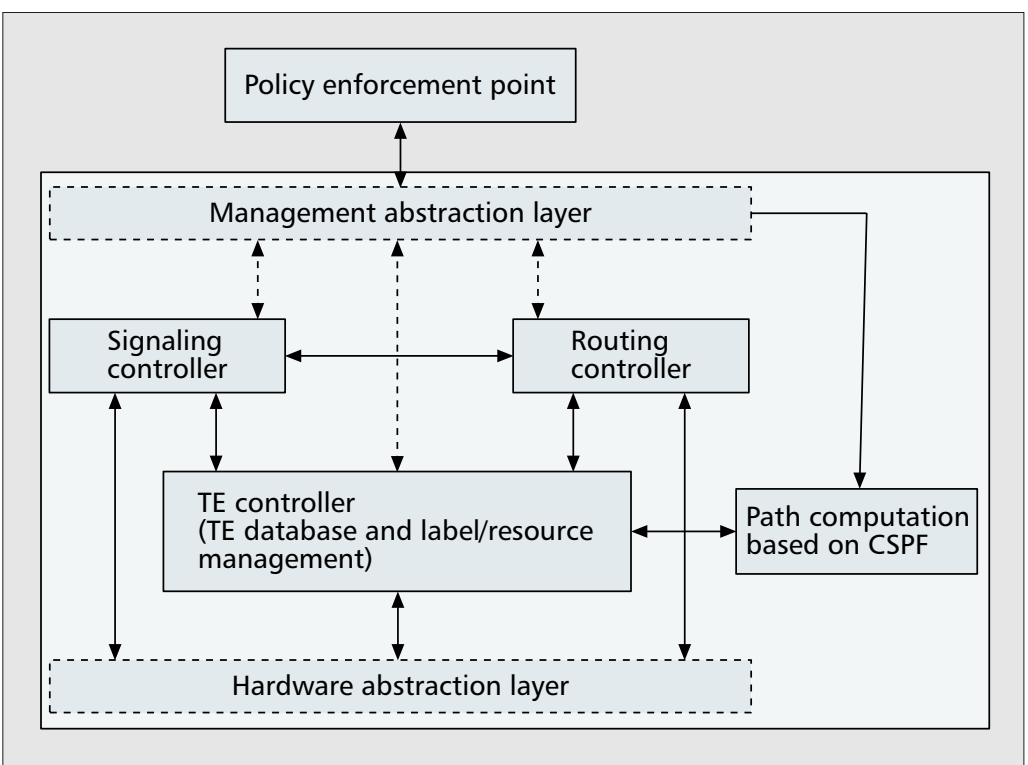

Figure 3. Internal control plane elements connected to the PBM system.

\section{BIOGRAPHIES}

LOA ANDERSSON works as principal networking architect at Acreo $A B$ in internetworking architecture and systems design, primarily with the Acreo National Broadband Testbed, but also in supporting Nordic and international research institutions and networking companies. This includes working with companies' design and marketing organizations to develop product and market strategies. He has provided leadership in architectural development and product specification at several networking companies. He is currently leading an architectural requirement and test activity for multiservice metro networks in the Nordic region. He is a member of the technical program committee of the MPLS World conference, Paris, France, and the Annual MPLS Conference, Washington, DC. He has been a speaker and/or chair at IP-dagarna, Stockholm, Sweden, 1999-2003; Telekomdagarna, Sweden, 2000, 2002, and 2003; MPLS World, Paris, France, 2001-2004; and MPLS 2001-200-4, Washington, DC. He has long experience working in standards organizations, and is also an active participant in the IETF and several industrial fora. He is currently co-chair of the MPLS and L2VPN working groups. He is a co-author of key MPLS, L2VPN, L3VPN, and GMPLS specifications. He has filed several patents in the area of networking. He is since March 2005 a member of the Internet Architecture Board. He graduated in chemistry in 1970 and, apart from his engineering degree, has also studied social anthropology and ethnology at Stockholm University.

BELA BERDE [M] (bela.berde@alcatel.fr) works, since 2000, for the Packet Transport Networking (PTI) group of Alcatel Research \& Innovation (R\&l), Marcoussis, France. Previously, he was a scientific (research) staff member at the French Aerospace Research Center (ONERA). He received his Ph.D. in applied mathematics from Universite Pierre et Marie Curie, Paris, France. He is currently involved in the design and development of new technologies for GMPLS-enabled multilayer networks. His current research interests are the design of network control and management solutions for communication systems. He is involved in different internal research programs and external development activities in liaison with academia, national operators, and industrials. $\mathrm{He}$ is also serving as reviewer and coordinator for the European CELTIC initiative.

DIDIER COLLE received an M. Sc. degree in electrotechnical engineering (option, communications) from Ghent University in 1997. Since then, he has been working at the same university as a researcher in the Department of Information Technology (INTEC). He is part of the research group INTEC Broadband Communication Networks (IBCN) headed by Prof. Piet Demeester. His research led to a Ph.D. degree in February 2002. He was granted a postdoctoral scholarship from the Institute for the Promotion of Innovation through Science and Technology in Flanders (IWT-Vlaanderen) for 
the period 2003-2004. His research deals with design and planning of communication networks. This work focuses on optical transport networks, to support the next-generation Internet. Until now, he has actively been involved in several IST projects (LION, OPTIMIST, DAVID, STOLAS, NOBEL and LASAGNE), COST actions 266 and 291, and the ITEA/IWT TBONES project. His work has been published in more than 100 scientific publications in international conferences and journals.

TIBOR CINKLER [M'95] (cinkler@tmit.bme.hu) received M.Sc. (1994) and Ph.D. (1999) degrees from Budapest University of Technology and Economics, Hungary, where he is currently an associate professor in the Department of Telecommunications and Media Informatics. His research interests focus on routing, design, configuration, dimensioning, $T E$, and resilience of IP, Ethernet, MPLS, ATM, ngSDH/SONET, and particularly WR-DWDM-based multilayer (GMPLS/ASTN) networks. He has been involved in a few related European and Hungarian projects (IP NOBEL, NoE e-Photon/ONe, CELTIC PROMISE, COST266, COST291, COST293, etc.) and is a member of ONDM, DRCN, ICC, Opticomm/BroadNets, EUNICE, and ICOCN Program Committees. He is the author of over 80 scientific publications and three patents.

JUAN FERNANDEZ-PALACIOS received his Telecommunications Engineer degree from Polytechnic University of Valencia, Spain, in June 2000, where he conduced his final project on the design of wavelength converters. He joined Telefonica I+D in September 2000, where he has been working on the evaluation of new transport technologies for access and core networks. He has participated in several European research projects such as Eurescom P1014 TWIN, IST DAVID, MUSE, NOBEL, and ePhoton/ONE as well as other Telefonica internal projects related to the development of optical networks in Spain and Latin America.
MONIKA JÄGER [M] (monika.jaeger@t-systems.com) has been a senior scientist and project leader in the Network Architecture Department of T-Systems International $\mathrm{GmbH}$ Technologiezentrum, Berlin, Germany, since 1998. She held previous positions with the Fraunhofer Institute for Open Communication Systems (FOKUS) and DeTeWe in Berlin. In 1992, she graduated in electrical engineering from Technische Universität München, Germany. Her current research interests are in the area of optical transport network design. She is involved in the European IST project NOBEL.

LIEVEN LEVRAU [M] (Ilevrau@riverstonenet.com) received an M.Sc. degree in applied physics engineering (focus on photonics) from the Free University of Brussels in 1994, and he has studied avionics at the University of Ghent. He has participated in several European and national projects, such as NOBEL, Dynamo, and GSN. His current research interests are focused on design issues related to multilayer network control architectures for core and metro networks. He is currently employed by Riverstone Networks as a technology consultant engineer.

MARTIN VIGOUREUX [M] (martin.vigoureux@alcatel.fr) received an $\mathrm{M}$.Sc. degree in high frequency electronics in 2000. He has been working since at Alcatel Research and Innovation. His current areas of interest are focused on GMPLS and its applicability to integrated systems architectures and advanced networks environments. He has coauthored publications on multiregion networks, including submissions to the CCAMP Working Group of the IETF, as well as others on policy-based management. He is also responsible for development of a GMPLS-enabled and flow-based network simulator as well as for a multilayer traffic engineering software.

\section{IEee Communications Magazine - Call for Papers Network \& Service Management Series}

IEEE Communications Magazine announces the creation of a new series on Network and Service Management. The series will be published twice a year, in April and October, with the first issue planned for October 2005. It intends to provide articles on the latest developments in this well-established and thriving discipline. Published articles are expected to highlight recent research achievements in this field and provide insight into theoretical and practical issues related to the evolution of network and service management from different perspectives. The series will provide a forum for the publication of both academic and industrial research, addressing the state of the art, theory and practice in network and service management. Both original research and review papers are welcome, in the style expected for IEEE Communications Magazine. Articles should be of tutorial nature, written in a style comprehensible to readers outside the speciality of Network and Service Management. This series therefore complements the newly established IEEE Electronic Transactions on Network \& Service Management (eTNSM). General areas include but are not limited to:

* Management models, architectures and frameworks

* Service provisioning, reliability and quality assurance

* Management functions

* Management standards, technologies and platforms

* Management policies

* Applications, case studies and experiences

The above list is not exhaustive, with submissions related to interesting ideas broadly related to network and service management encouraged.

IEEE Communications Magazine is read by tens of thousands of readers from both academia and industry. The magazine has also been ranked the number one telecommunications journal according to the ISI citation database for year 2000, and the number three for year 2001. The published papers will also be available on-line through Communications Magazine Interactive, the WWW edition of the magazine. Details about IEEE Communications Magazine can be found at http://www.comsoc.org/ci/.

\section{SCHEDULE FOR THE FIRST ISSUE}

Manuscripts due: March 30, 2005

Acceptance notification: June 30, 2005

Manuscripts to publisher: July 30, 2005

Publication date: October 2005

\section{SERIES EDITORS}

Prof. George Pavlou

Center for Communication Systems Research

Dept. of Electronic Engineering

University of Surrey

Guilford, Surrey GU2 7XH, UK.

e-mail: G.Pavlou@eim.surrey.ac.uk
Dr. Aiko Pras

Center for Telematics and Information Technology

Dept. of Electrical Engineering, Mathematics and Computer Science University of Twente

P.O. Box 217, 7500 AE Enschede, The Netherlands.

e-mail: a.pras@utwente.nl 